\title{
Coordinated Experiments for Projections of Regional Climate Change
}

\author{
Filippo Giorgi $^{1,2} \cdot$ William J. Gutowski ${ }^{1,2}$
}

Published online: 5 September 2016

(C) Springer International Publishing AG 2016

\begin{abstract}
We review coordinated efforts for producing regional climate projections through dynamical and statistical downscaling tools driven by global climate model output. Such projections are affected by multiple sources of uncertainty both at the global model and at the downscaling levels. The characterization of these uncertainties and the production of robust regional to local projections for use in impact studies require the completion of properly designed large ensembles of experiments. Toward this purpose, several regional coordinated efforts have been conducted in the past, particularly involving regional climate models, but because of the lack of a common experiment protocol, the transfer of know-how across them has been difficult. This problem is being addressed in the Coordinated Regional Downscaling Experiment (CORDEX), a framework designed to produce the next generation of worldwide high-resolution regional climate projections through a fully coordinated experiment protocol.
\end{abstract}

Keywords Regional climate projection · Downscaling · Regional climate modeling $\cdot$ Statistical downscaling

This article is part of the Topical Collection on Advances in Modeling

Filippo Giorgi

giorgi@ictp.it

1 Abdus Salam International Centre for Theoretical Physics (ICTP), Trieste, Italy

2 Iowa State University, Ames, IA, USA

\section{Background and Introduction}

The issue of regional climate projection is central to the provision of climate change information needed in vulnerability, impacts, and adaptation (VIA) assessment studies. The primary tools available today to generate climate projections are coupled global climate models (GCMs); however, their horizontal spatial resolution, currently order of $1^{\circ}$, is too coarse for many needs of VIA applications. As a consequence, different regional climate downscaling (RCD) techniques are used to produce climate change information at sub-GCM resolution scales [1]. These techniques include the use of limited area regional climate models (RCMs [2]), empirical statistical downscaling (ESD [3]) methods, and variable and high-resolution atmospheric GCMs (VARGCMs [4] and HIRGCM [5], respectively).

All RCD techniques use as input large-scale variables produced by GCMs or reanalyses of observations to generate spatially refined climate information. For the case of RCMs, the models need initial and time-dependent atmospheric boundary conditions (typically wind, temperature, moisture, and surface pressure) as well as sea surface temperature (SST) and, when relevant, sea ice conditions (e.g., sea ice extent and surface temperature). VARGCMs and HIRGCMs are generally forced only by externally prescribed SSTs, while different ESD methods may require different large scale forcing inputs.

The process of producing future regional climate projections through downscaling is affected by multiple sources of uncertainty which may compound in a cascade process [6]. On the GCM side, the primary uncertainty sources are associated with future greenhouse gas (GHG) and aerosol emission/concentration scenarios (or "scenario" uncertainty, usually sampled by simulating a range of GHG/aerosol concentration scenarios); the response of different models to the same GHG/aerosol forcing, often also dependent on the model systematic errors 
(or "structural" uncertainty, usually sampled by using ensembles of models); and the internal variability of the climate system (or "internal variability" uncertainty, usually sampled by completing different realizations of the same scenario run). These uncertainties are then transmitted to the downscaled projections through the provision of the GCM forcings and are compounded by analogous uncertainty sources in the downscaling itself (e.g., RCM structural and internal variability uncertainty) and by the uncertainty associated with the use of different downscaling methods (e.g., RCMs vs. ESD). In addition, climate variability increases at finer scales [7] which makes the extraction of forced climate change signals from the underlying natural variability more difficult.

The characterization of the robustness and uncertainty underlying regional climate projections, which is paramount to the proper use of such projections in VIA applications, requires the use of multi-model, multi-method approaches. This realization has given impetus to the development of coordinated programs sharing common protocols in order to facilitate the analysis, intercomparison, and synthesis of different simulations. On the GCM side, for example, the Coupled Model Intercomparison Project (CMIP) series has provided a common framework toward the production of coordinated GCM global projections (e.g., CMIP3 [8], CMIP5 [9]). The CMIP programs have led to a tremendous advancement in the understanding of models and climate change issues, generating datasets that have been used by a large and growing scientific community.

On the downscaling side, a number of regional RCD intercomparison projects have been implemented, which have certainly led to advances in the knowledge of regional modeling tools and projections. However, because of differences in simulation designs, it has been difficult to transfer know-how across regional settings. This problem was already recognized in the mid-2000s [10], but only with the inception of the Coordinated Regional Downscaling Experiment (CORDEX $[11,12])$, has a common experiment protocol been developed for generating large RCD-based ensembles of climate projections over regions worldwide. CORDEX represents a fundamental advance in the coordination of RCD research and provides the main current framework for RCD activities related to regional climate change science.

In this paper, we first discuss the rationale behind the need for coordinated RCD projects ("Why RCD-Coordinated Projects?" section), we then provide a brief review of past regional projects (“A Brief Review of Coordinated Regional Projection Projects Prior to the CORDEX Program" section), and finally we discuss the main developments within the CORDEX program ("The Coordinated Regional Downscaling Experiment" section). We do not present a review of downscaling techniques and issues, since a number of review papers are already available in the literature, which any user of RCD techniques is advised to read ([1-5, 13-21]).

\section{Why RCD-Coordinated Projects?}

By its very nature, regional downscaling lends itself to fragmentation, as different communities are often interested in different problems or have varied needs and technical requirements. However, the use of common experimental protocols facilitates the analysis and intercomparison of results and offers clear added opportunities to better understand models, processes, and projections. On the one hand, large ensembles covering the different dimensions of the uncertainty space (e.g., scenario, structural, and internal variability) are needed to properly characterize the full uncertainty range in projections [22]. On the other hand, ensembles are necessary to provide robustness and credibility to the projections through different lines of evidence, such as intermodel agreement, identification of underlying processes common to the models, and consistency with observed trends.

A further important role of coordinated multi-model experiments is the identification of common systematic errors. For example, it has been shown that most RCMs tend to underestimate precipitation over the La Plata basin, when forced by either GCM or reanalysis fields [23]. As another example, various generations of RCMs for the European region exhibit a tendency to be excessively dry during the summer over southeastern Europe [24]. Similarly, a common systematic bias in RCMs for North America is the underestimation of winter topographic precipitation over the southeastern USA in conjunction with excessive rain on the lee side of the western US ranges [25]. Since coordinated experiments have shown that such errors are shared by most models, it is likely that they are symptomatic of basic model deficiencies in simulating underlying relevant processes and can therefore significantly affect regional projections [26].

By the same token, multi-model experiments can highlight in robust ways the added value of downscaled information compared to the driving GCMs, an issue that is central to the downscaling exercise [27]. A typical example is the added value of high-resolution RCMs in simulating topographically induced precipitation patterns along with daily precipitation intensity distributions and extremes [28, 29], also within the context of climate change simulations [30].

An important conclusion found through the use of coordinated multi-model RCD experiments is the substantial contribution of intermodel RCM structural uncertainty to the overall uncertainty of regional projections. This is illustrated in Fig. 1, derived from the European project PRUDENCE [31], which shows the fractional contributions to the total projection uncertainty for different variables, of the GCM and RCM structural uncertainties, the internal GCM variability, and the emission scenario uncertainty. Even though the full uncertainty ranges were only partially covered in the PRUDENCE ensemble, it can be seen that for variables such as winter temperature and precipitation, which are mostly driven by large-scale processes, 
Fig. 1 Fractional contributions of different sources of uncertainty to the full uncertainty range in the PRUDENCE projections (20712100 vs. $1960-1990$; high-end A2 scenario) averaged over the European region for different variables. $T-D J F$ is winter temperature; T-JJA is summer temperature; $P-D J F$ is winter precipitation; $P-J J A$ is summer precipitation. " $G C M$ " indicates the GCM structural uncertainty (four GCMs), "Variability" the internal variability uncertainty (three realizations for one GCM/ RCM pair), "Scenario" the scenario uncertainty (high-end and low-end scenarios), " $R C M$ " the RCM structural uncertainty (nine RCMs). The data are adapted from [31]
T-DJF

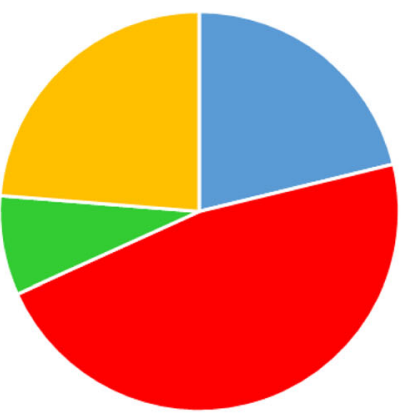

P-DJF

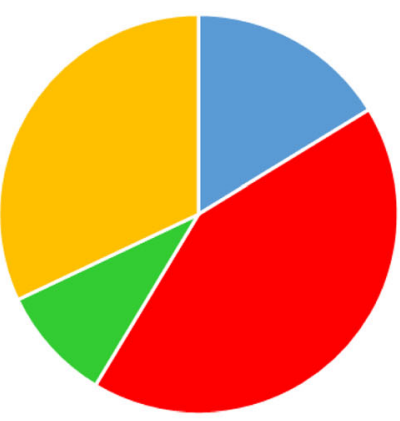

T-JJA

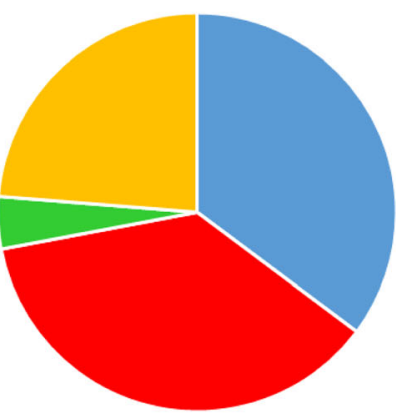

P-JJA

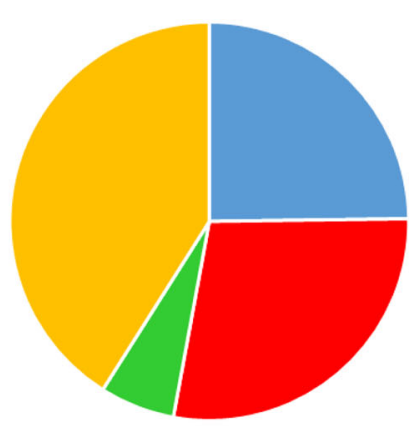

- Scenario $\backsim$ GCM $\backsim$ Variability $\backsim \mathbf{R C M}$

the contribution of RCM structural uncertainty is relatively small. In contrast, for summer precipitation, which is more related to local convective processes, the RCM and GCM structural uncertainty contributions are comparable. This conclusion is not obvious, since it challenges the notion that the boundary forcing dominates the RCM response at broad subcontinental scales, at least in contexts for which the RCM representation of local processes (e.g., summer convection) is important. A similar conclusion was found in the AMMA project over West Africa [32], the North America Regional Climate Change Assessment Program (NARCCAP) project over North America [33], and the CORDEX project over South America [34], where different RCMs or RCM configurations (e.g., physics schemes) provided quite different subcontinental scale precipitation change responses even when driven by the same GCM.

In fact, often the climate response (e.g., precipitation change) in RCMs vs. the driving GCMs can be different not only in magnitude but also in sign $[28,33]$. While in some cases this can be clearly attributed to local forcings (e.g., finescale topographic features [28]), in others it may simply be related to the different physics representations in the models. Similarly, the change patterns calculated by different RCD techniques (e.g., RCM vs. ESD) have been often shown to substantially vary across methods [3]. These results highlight that in order to fully characterize uncertainties and arrive at the most robust possible conclusions, it is essential to assess and use approaches based on multi RCD models and techniques.

The design of a coordinated experiment requires a common and internally consistent simulation protocol in order to maximize the comparability of results. This is especially important when dealing with downscaling techniques. For example, RCM simulations are sensitive to model resolution and domain specifications [35], and often different physics options within the same model system provide better performances in different regions [36]. Similarly, ESD techniques can be highly variable in terms of their input, output, and assumptions, and the observations necessary to calibrate them can be of varied quality and density across regions [3, 20,21].

In addition, the analysis of RCD models needs to be based on multiple criteria. Common performance metrics across different regions can provide a measure of systematic model behaviors. However, regionally specific and process-based analyses provide additional ways to evaluate the model performance for different regional contexts and thus yield a more thorough assessment of the RCD ensemble.

Another critical issue in the experiment design is the choice of $\mathrm{GCM} / \mathrm{RCD}$ matrix used for the downscaled projections, especially if this matrix is sparse. Often, the selection of driving GCMs has been based on the availability of the proper set 
of time varying GCM output variables for downscaling ("ensemble of opportunity"). However, the GCM selection should actually be based on well-designed criteria, for example, the model performance over the target region or globally, and the representativeness of the driving models of the full GCM range in regional responses [37, 38]. Clearly, the extraction of robust regional information from multiple and varied sources of different quality is a research challenge in itself, which requires a careful design of simulation strategies.

\section{A Brief Review of Coordinated Regional Projection Projects Prior to the CORDEX Program}

Although RCD tools have been essentially used for all land regions of the world, to date, only a limited number of coordinated projects have been carried out based on the use of RCMs or ESD techniques. A set of major RCM coordinated projects is reported in Table 1 . The different projects generally utilized different experiment designs (e.g., simulation length or scenarios), but they mostly shared a basic approach consisting of a model evaluation stream using reanalyses of observations to drive the RCMs and, for the projects that included future climate projections, a model projection stream in which the RCMs were driven by different GCMs.

The first attempt at coordinating RCM simulations under a common framework was PIRCS [39], in which ensembles of up to $13 \mathrm{RCMs}$ were run for the two extreme cases of the summer 1988 (drought) and 1993 (flood) over the continental USA with a grid spacing of $50 \mathrm{~km}$. Even if limited in length, these experiments emphasized the usefulness of using model ensembles to expose common behaviors despite differences in convective and land surface parameterizations. Also covering the continental USA was the more recent project NARCCAP
[40], in which a simulation matrix was (partially) populated, comprising six RCMs and four driving GCMs under one GHG emission scenario. The NARCCAP program resulted in the production of relatively large projection ensembles that are widely used for a range of VIA applications [33].

The European research community has been extremely active in regional climate modeling. A series of RCM projects funded by the European Commission were completed in the late 1990s (MERCURE [41]), mid-2000s (PRUDENCE [42]), and late 2000s (ENSEMBLES [43]). In particular, PRUDENCE was a landmark project for RCM research in that for the first time a relatively well-populated experiment matrix was completed for present day and future climate 30year time slices (grid spacing of $50 \mathrm{~km}$ ), including four GCMs, nine RCMs, and two GHG emission scenarios. This matrix allowed a robust characterization of both systematic model errors [24] and future changes in climatologies, variability and extremes for the European region [44, 45], including the assessment of different sources of projection uncertainties [31]. In addition, PRUDENCE encompassed a series of VIA assessment studies that allowed an evaluation of the application value of RCM-derived information [46]. The framework of PRUDENCE was then augmented by the subsequent project ENSEMBLES in which a larger and more coordinated GCM-RCM matrix was completed with models at higher horizontal resolution (grid spacing of $25 \mathrm{~km}$ ) and longer simulations (full twenty-first century transient runs), along with a more detailed exploration of model errors, improvements, and weighting techniques [43, 47-49].

Coordinated multi-RCM projects for tropical monsoon regions include AMMA for West Africa [32, 50], RMIP for East Asia [51], and CLARIS(-LPB) for South America [52]. In all cases, the RCMs were able to reproduce the basic monsoon circulation and seasonal precipitation patterns, however with
Table 1 Set of major regional coordinated RCM projects, prior to the inception of CORDEX

\begin{tabular}{lll}
\hline Acronym & Name & Region \\
\hline PIRCS [38] & Project to Intercompare Regional Climate Simulations & North America \\
NARCCAP & North America Regional Climate Change Assessment Program & North America \\
[39] & & \\
MERCURE & Modeling European Regional Climate, Understanding and reducing & Europe \\
[40] & Errors & \\
PRUDENCE & Prediction of Regional Scenarios and Uncertainties for Defining & Europe \\
[41] & European Climate Change Risks and Effects & Europe \\
ENSEMBLES & Ensemble-based Predictions of Climate Changes and their Impacts & \\
[42] & & West Africa \\
AMMA [46] & African Monsoon Multidisciplinary Analysis & East Asia \\
RMIP [47] & Regional Model Intercomparison Project & South America \\
CLARIS (- & A Europe-South America Network for Climate Change Assessment and & \\
LPB) [48] & Impact Studies (-La Plata basin) & Arctic \\
ARCMIP [50] & Arctic Regional Climate Model Intercomparison Project & Baltic Sea \\
BALTEX [52] & Baltic Sea Experiment & drainage \\
& & basin \\
\hline
\end{tabular}


different levels of performance across models and some common systematic errors [53]. An important conclusion of these projects was that over tropical domains the internal RCM physics (especially convective and land surface schemes) had a substantial role in determining the model performance and climate change response with respect to the lateral boundary forcing [32, 53].

A further RCM intercomparison project to highlight is ARCMIP [54], in which an ensemble of RCMs, both atmosphere only and coupled atmosphere-ocean-sea ice, were used to simulate the climate of the Arctic region. This is an especially challenging region for climate models since many processes and circulations in the Arctic are poorly understood and their representation in climate models depends strongly on the physical schemes utilized. Specifically, in ARCMIP, the RCMs were evaluated against an intensive observation campaign that occurred in 1997-1998 and provided the grounds for a detailed and multi-variable model assessment [55].

The development of coupled atmosphere-land-ocean $\mathrm{RCMs}$ is one of the main areas of strong development in regional modeling research [2], and a major contribution to this development was given by the BALTEX project [56], in which coupled RCM systems were developed to study in an integrated way the water and energy cycles, climate change, variability and extremes, and impacts on the biogeochemical cycle of the Baltic Sea drainage basin. Coupled atmosphereland-ocean RCM systems have been developed also for the Mediterranean Sea [57, 58], the Caspian Sea [59], and the South Asia [60].

In contrast to the substantial development of RCM multimodel programs, ESD development has been more fragmented. There have been some ESD intercomparison studies [61-64] but often ESD efforts have specific goals, and partly as a consequence, many varied types of applications (e.g., [65-68]). The rich variety of methods and applications demonstrates the flexibility of ESD but has also hindered the development of coordinated multi-method programs. Some studies have intercompared ESD and RCM performances (e.g., [69-72]), and the results have generally suggested both advantages and limitations of the two approaches. Concerning VARGCM and HIRGCM downscaling, several systems are today available [4], but only limited coordinated intercomparison efforts for present day climate conditions have been conducted to date $[73,74]$.

\section{The Coordinated Regional Downscaling Experiment}

The Coordinated Regional Downscaling Experiment (CORDEX) program was launched under the auspices of the World Climate Research Program (WCRP) with the vision to advance regional climate research and application through downscaling $[11,12]$. Specifically, among the CORDEX goals, we highlight the assessment and improvement of RCD models and techniques and the enhanced understanding of regional climate change processes, projections, and uncertainties. CORDEX is based on the production of large ensembles of multi-technique and multi-model simulations following a common simulation protocol across regions worldwide in order to facilitate intermodel, cross-technique, and crossdomain analyses.

The CORDEX phase I experiment framework $[11,12]$ included a model evaluation stream based on forcing data from the ERA-interim reanalysis of observations [75] and a model projection stream based on downscaling multiple CMIP5 GCMs by multiple RCMs and ESD techniques over a set of 14 domains covering essentially all land areas of the world (www.cordex.org). In addition, the project is also intended to encompass available VARGCM and HIRGCM models. For the RCMs, a model grid spacing of $50 \mathrm{~km}$ was adopted as baseline in order to enhance participation by a wide community, and the Africa domain was selected as the highest priority because of the pronounced vulnerability of this continent to global warming and the lack of local infrastructure to carry out climate projections.

The CORDEX phase I activities have resulted in the completion of present day and/or projection ensembles of differing sizes for most domains, including Africa [76], Europe [77], the Mediterranean [78], the Arctic [79], South Asia [80], East Asia [81], Southeast Asia [82], South America [83], North America [84], Central America [85], the Middle-East North Africa (MENA [86]), and Central Asia [87]. Of particular relevance are the EURO-CORDEX [77] and MEDCORDEX [78] initiatives. In the former, large multi-model ensembles have been completed at two nominal resolutions, $0.44^{\circ}$ and $0.11^{\circ}$, resulting in an unprecedented set of highresolution projections for use in VIA studies. Conversely, Med-CORDEX has focused more on the development and use of coupled Regional Earth System Models for the Mediterranean basin including atmosphere, ocean, river, and aerosol components applied to the assessment of the interactions across these components in modifying regional climate change signals. For example, it was shown that both ocean coupling [88] and aerosol forcings [89] can have substantial impacts on the climate patterns over the Mediterranean basin.

Figure 2 shows an example of the simulation of fall precipitation over the European Alps in an ensemble of EUROCORDEX and MED-CORDEX GCMs, $0.44^{\circ}$ and $0.11^{\circ} \mathrm{km}$ resolution RCMs for a present day period (1976-2005) along with the corresponding change patterns for the end of the twenty-first century (2071-2100) under the RCP8.5 emission scenario [90]. Comparison with a high-resolution observation dataset [91] first illustrates the added value of high-resolution RCMs in capturing the spatial detail of topographically induced precipitation (top panels). The bottom panels then show how the fine scale representation of Alpine topography can 


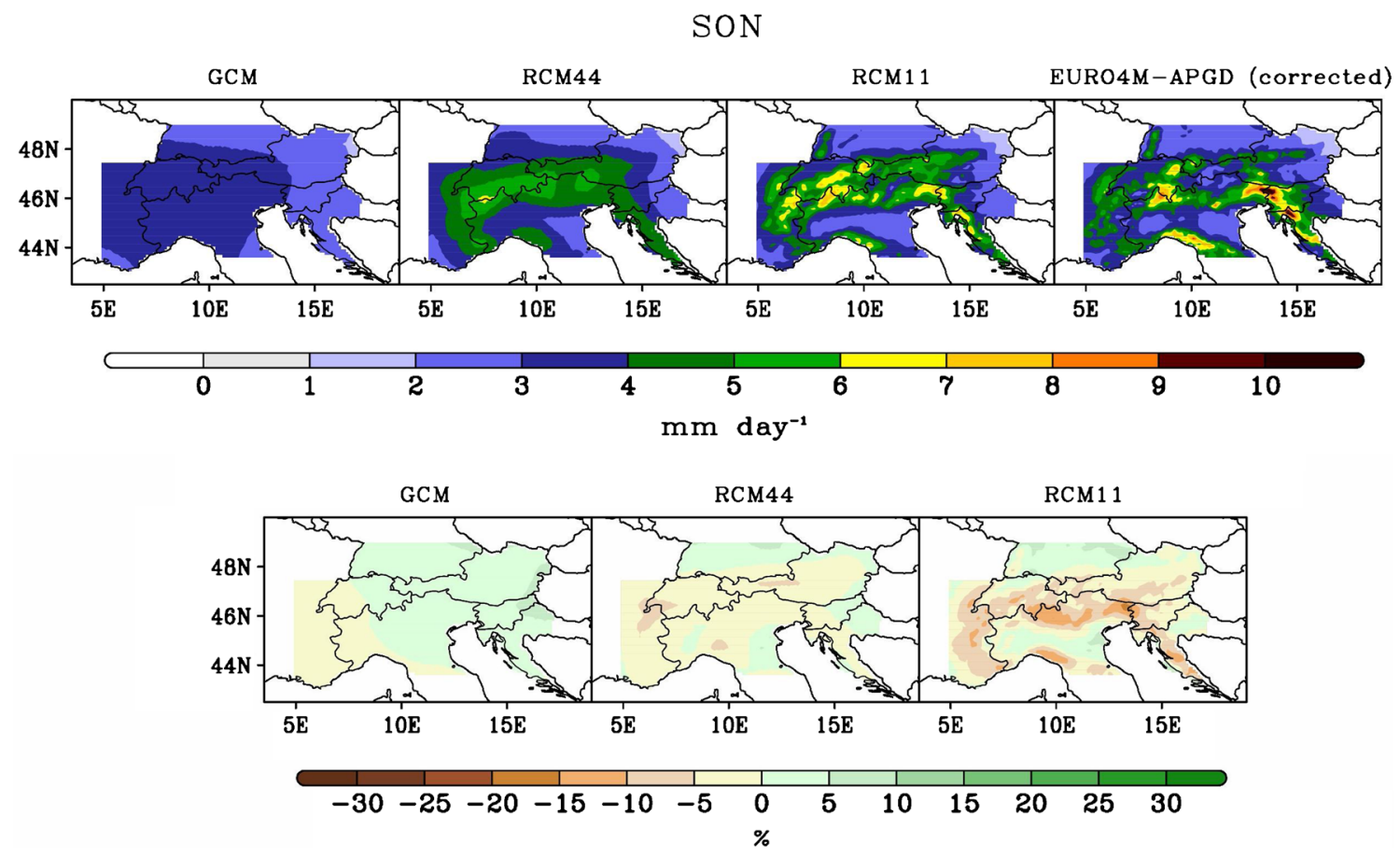

Fig. 2 Fall (September-October-November or $S O N$ ) precipitation in an ensemble of four driving GCMs and six nested RCMs run at resolutions of $0.11^{\circ}$ and $0.44^{\circ}$ (RCM44 and RCM11, respectively) over the Alpine region in the EURO-CORDEX and MED-CORDEX initiatives. The top panels show the mean precipitation $(\mathrm{mm} /$ day) for the present day period (1975-2004) and compare the model data with a high-resolution observation dataset [87] (in the last panel to the right, observations include a gauge undercatch correction as described in [28]). The bottom panels show the corresponding mean precipitation change (units of $\%$ of present day values) for the period 2070-2099 with respect to 1975-2004 under the RCP8.5 greenhouse gas concentration scenario [86]. The figure is adapted from [28] substantially affect the precipitation change signal (compared to the driving GCMs) both in sign and in magnitude. Other studies have then shown the importance of resolution for the simulation of precipitation intensity distributions and extremes [28, 92].

In addition to highlighting the role of resolution for regional projections, the CORDEX phase I activities emphasized the importance of large multi-model ensembles, since for example, the changes in phenomena such as monsoon precipitation $[32,80]$ and tropical storms [85] are found to strongly depend on the model physics configurations. Unfortunately, however, the number of simulations available for different CORDEX regions is highly variable and this inhomogeneity has made it difficult to carry out homogenous assessments across regions.

These considerations have provided the main guidelines for the ongoing discussions on the design of the CORDEX phase II framework [2], which is currently planned to encompass two components. The first, referred to as Common Regional Experiment framework (or CORDEX-CORE), envisions the completion of a common minimum ensemble of projections for all CORDEX domains by a core set of RCMs downscaling a core set of GCMs and scenarios at nominal resolutions in the range of 10-25 km. This baseline common ensemble would then be incrementally populated over the different regions by additional available experiments and by information derived from ESD methods. While the details of this CORDEX-CORE framework are still being discussed, this approach would guarantee the availability of a homogeneous core ensemble across all CORDEX regions.

The second component of the CORDEX phase II framework is the concept of Flagship Pilot Studies (FPSs). FPSs are intended to be frontline research projects aimed at addressing in detail targeted science questions relevant to specific regional settings. A typical example is the downscaling to horizontal nominal resolutions of few kilometers through the use of convection-permitting non-hydrostatic models [93, 94], which is one of the primary areas of future development in RCM research [2]. Other examples would be the rigorous comparison of projections by different downscaling or post-processing techniques (e.g., RCMs, ESD and bias correction [95]) or the assessment of regional to local forcings, such as land use change and aerosols. While it would be difficult to carry out these studies for the continental scale CORDEX domains, they can be addressed within specific targeted regions. FPSs are expected to be bottom-up initiatives, with proposals submitted by regional communities and eventually endorsed by CORDEX after an evaluation process (www.cordex.org).

Complementing these phase II activities is the development of a CORDEX ESD framework. To date, this has occurred through a series of workshops [96] that have designed a 
program aimed at evaluating strengths and shortcomings of a variety of ESD methods themselves and in comparison with dynamical downscaling. The workshops developed the protocols for an initial intercomparison experiment focused on a portion of South America with relatively high-density and high-quality observations to calibrate and assess the ESD output. The primary objectives for this initial case are to delineate the relative skills of different statistical downscaling approaches when applied to a common source of predictors and predictands and to assess the added value that ESD can provide from coarse scale predictor fields taken from GCMs. As the ESD framework progresses, the ESD efforts will have a foundation for expanding to blend evaluations of both dynamical and statistical downscaling.

\section{Final Considerations}

In this paper, we have reviewed the issue of coordinated efforts to produce regional climate projections through RCD techniques. The call for large multi-model and multi-method ensemble approaches stems from the need of producing robust regional climate information based on multiple lines of evidence and of properly characterizing the different sources of uncertainty in regional projections. Until recent years, individual regional coordinated projects have provided valuable information on the behavior of ensembles of downscaled projections; however, these efforts lacked cross-project coordination in terms of simulation and analysis protocols.

The completion and application of regional downscaled projections is a fast growing area of research by a wide and varied community, so that it is important to fully understand the value, limitations, and uncertainties of such projections. Coordinated experiments thus play a fundamental role in this regard by providing a rigorous framework to assess $\mathrm{RCD}$ models and regional climate projections. Climate change information for VIA application can be derived by a multitude of sources, such as GCMs, RCMs, ESD, and post-processing techniques (bias correction), often diverging not only in magnitude but also in sign and affected by the presence of model systematic errors. The distillation of robust information from these multiple sources hinges upon the availability of large ensembles based on carefully designed simulation protocols and fully evaluated models, which can optimally be obtained only through the development of coordinated multi-model experiments.

The CORDEX program is designed to address these problems and provide a common platform for coordinating regional downscaling activities in order to produce more homogenous and quality controlled regional projection information and to facilitate transfer of know-how across regions and with the VIA community. As a result of its first phase activities, CORDEX has steadily grown in the last few years, as demonstrated for example in two major Pan-CORDEX conferences (November 2013 in Brussels and May 2016 in Stockholm) with more than 400 abstracts submitted. As detailed in the previous section, the discussions on the design of the second phase CORDEX activities are currently under way.

Acknowledgments We would like to thank C. Torma for the help in producing the two figures used in the paper. We also thank two anonymous reviewers for their helpful comments and suggestions.

\section{Compliance with Ethical Standards}

Conflict of Interest On behalf of all authors, the corresponding author states that there is no conflict of interest.

\section{References}

1. Giorgi $\mathrm{F}$ et al. Regional climate information - evaluation and projections. Chapter 10 of Climate change 2001; The scientific basis. In: Houghton JD et al., editors. Contribution of Working Group I to the Third Assessment Report of the Intergovernmental Panel on Climate Change (IPCC). Cambridge: Cambridge University Press; 2001. p. 583-638.

2. Giorgi F, Gutowski Jr WJ. Regional dynamical downscaling and the CORDEX initiative. Annu Rev Environ Resour. 2015;40:467-90.

3. Maraun D et al. Precipitation downscaling under climate change: recent developments to bridge the gap between dynamical models and the end user. Rev Geophys. 2010;48:RG303.

4. McGregor JL. Recent developments in variable-resolution global climate modeling. Clim Chang. 2015;129:369-80.

5. Cubasch U, Waszkewitz J, Hegerl G, Perlwitz J. Regional climate changes as simulated in time-slice experiments. Clim Chang. 1995;31:273-304.

6. Giorgi F. Climate change prediction. Clim Chang. 2005;73:239-65.

7. Giorgi F. Dependence of surface climate interannual variability on spatial scale. Geophys Res Lett. 2002;29:2101.

8. Meehl GA et al. The WCRP CMIP3 multimodel dataset: a new era in climate change research. Bull Am Meteorol Soc. 2007;88:1383-94.

9. Taylor KE, Stouffer RJ, Meehl GA. An overview of CMIP5 and the experiment design. Bull Am Meteorol Soc. 2012;93:485-98.

10. Takle ES, Roads J, Rockel B, Gutowski Jr WJ. Transferability intercomparison: an opportunity for new insight on the global water cycle and energy budget. Bull Am Meteorol Soc. 2007;88:375-89.

11. Giorgi F, Jones C, Asrar G. Addressing climate information needs at the regional level: the CORDEX framework. WMO Bull. 2009;58: $175-83$.

12. Jones C, Giorgi F, Asrar G. The Coordinated Regional Downscaling Experiment. An international downscaling link to CMIP5. CLIVAR Exchanges. 2011;16:34-40.

13. Wang $\mathrm{Y}$ et al. Regional climate modeling: progress, challenges and prospects. J Meteorol Soc Jpn. 2004;82:1599-628.

14. Giorgi F. Regional climate modeling: status and perspectives. J Phys IV. 2006;139:101-18.

15. Laprise R. Regional climate modeling. J Comput Phys. 2008;227: 3641-66.

16. Rummukainen M. State-of-the-art with regional climate models. WIRES Climate Change. 2010;1:82-96.

17. Arritt RW, Rummukainen M. Challenges in regional scale climate modeling. Bull Am Meteorol Soc. 2011;92:365-8. 
18. Rockel B. The regional downscaling approach: a brief history and recent advances. Current Climate Change Reports. 2015;1:22-9.

19. Rummukainen $\mathrm{M}$ et al. Twentyfirst century challenges in regional climate modelling. Bull Am Meteorol Soc. 2015;96:ES135-8.

20. Hewitson B, Crane R. Climate downscaling: techniques and application. Clim Res. 1996;7:85-95.

21. Wilby RL, Wigley TML. Downscaling general circulation model output: a review of methods and limitations. Prog Phys Geogr. 1997;21:530-48.

22. Hawkins A, Sutton R. The potential to narrow uncertainty in regional climate predictions. Bull Am Meteorol Soc. 2009;90:1095-107.

23. Solman SA et al. Evaluation of an ensemble of regional climate model simulations over South America driven by the ERAinterim reanalysis: model performance and uncertainties. Clim Dyn. 2013;41:1139-57.

24. Jacob D et al. An intercomparison of regional climate models for Europe: design of the experiments and model performance. Clim Chang. 2007;81:31-52.

25. Kim J et al. Evaluation of the surface climatology over the conterminous United States in the North American Regional Climate Change Assessment Program hindcast experiment using a regional climate model evaluation system. J Clim. 2013;26:5698-715.

26. Boberg F, Christensen JH. Overestimation of Mediterranean summer temperature projections due to model deficiencies. Nat Clim Chang. 2012;2:433-6.

27. Rummukainen M. Added value in regional climate modeling. Wiley Interdiscip Rev Clim Change. 2016;7:145-59.

28. Torma C, Giorgi F, Coppola E. Added value of regional climate modeling over areas characterized by complex terrain - precipitation over the alps. J Geophys Res. 2015;120:3957-72.

29. Di Luca A, de Elia R, Laprise R. Potential for added value in precipitation simulated by high resolution nested regional climate models and observations. Clim Dyn. 2012;38:1229-47.

30. Giorgi $\mathrm{F}$ et al. Enhanced summer convective rain at Alpine high elevations in response to climate warming. Nat Geosci. 2016. doi:10.1038/NGEO2761.

31. Deque $\mathrm{M}$ et al. An intercomparison of regional climate simulations for Europe: assessing uncertainties in model projections. Clim Chang. 2007;81:53-70.

32. Paeth $\mathrm{H}$ et al. Progress in regional downscaling of West Africa precipitation. Atmos Sci Lett. 2011;12:116-22.

33. Mearns LO et al. Climate change projections of the North American Regional Climate Change Assessment Program (NARCCAP). Clim Chang. 2013;120:965-75.

34. Llopart-Pereira MP, Coppola E, Giorgi F., Da Rocha RP, Cuadra FP. Climate change impact on precipitation for the Amazon and $\mathrm{La}$ Plata basin. Clim Chang. 2014;125:111-125.

35. Larsen MAD, Thejll P, Christensen JH, Refsgaard JC, Jensen KH. On the role of domain size and resolution in the simulations with the HIRHAM regional climate model. Clim Dyn. 2013;40:2903-18.

36. Giorgi $\mathrm{F}$ et al. RegCM4: model description and preliminary tests of multiple CORDEX domains. Clim Res. 2012;52:7-29.

37. Elguindi N, Giorgi F, Turuncoglu UU. Assessment of CMIP5 global model simulations over the sub-set of CORDEX domains used in the phase I CREMA experiment. Clim Chang. 2014;125:7-21.

38. McSweeney CF, Jones RG, Lee RW, Rowell DP. Selecting CMIP5 GCMs for downscaling over multiple regions. Clim Dyn. 2015;44: 3237-60.

39. Takle ES et al. Project to Intercompare Regional Climate Simulations (PIRCS) description and initial results. J Geophys Res. 1999;104:19,443-61.

40. Mearns LO et al. The North American Regional Climate Change Assessment Program. Overview of Phase I results Bulletin of the American Meteorological Society. 2012;93:1337-62.
41. Christensen JH et al. Validation of present day regional climate simulations over Europe: LAM simulations with observed boundary conditions. Clim Dyn. 1997;13:489-506.

42. Christensen JH, Carter T, Rummukainen M, Amanatidis G. Evaluating the performance and utility of regional climate models: the PRUDENCE project. Clim Chang. 2007;81:1-6.

43. Van der Linden P, Mitchell JFB (co-eds). ENSEMBLES: climate change and its impacts. Summary of Research and Results of the ENSEMBLES Project. Met Office Hadley Centre, Fitzroy Road, Exeter EX1 3PB, UK; 2007. 260 pp.

44. Deque $\mathrm{M}$ et al. Global high resolution vs. limited area model climate change scenarios over Europe: quantifying confidence level from PRUDENCE results. Clim Dyn. 2005;25:653-70.

45. Beniston $\mathrm{M}$ et al. Future extreme events in European climate: an exploration in regional climate model projections. Clim Chang. 2007;71-95.

46. Fronzek S, Carter T. Assessing uncertainties in climate change impacts on resource potential for Europe based on projections from RCMs and GCMs. Clim Chang. 2007;81:357-71.

47. Deque $\mathrm{M}$ et al. The spread amongst ENSEMBLES regional scenarios: regional climate models, driving general circulation models and interannual variability. Clim Dyn. 2012;38:951-64.

48. Rauscher SA, Coppola E, Piani C, Giorgi F. Resolution effect of regional climate model simulation of precipitation over Europe. Part I: seasonal. Clim Dyn. 2010;35:685-711.

49. Christensen JH, Kjellstrom E, Giorgi F, Lenderink G, Rummukainen M. Assigning relative weights to regional climate models. Exploring the concept. Clim Res. 2010;44:179-94.

50. Ruti $\mathrm{P}$ et al. Modeling the West Africa climate system: systematic errors and future steps. Atmos Sci Lett. 2011;12:116-22.

51. $\mathrm{Fu} \mathrm{C}$ et al. Regional climate model intercomparison project for Asia. Bull Am Meteorol Soc. 2005;86:257-66.

52. Boulanger JP, Carrill AF, Sanchez E. CLARIS-La Plata Basin: regional hydroclimate variability, uncertainties and climate change scenarios. Climatic Research. 2016;68:93-4.

53. Solman SA. Systematic temperature and precipitation biases in the CLARIS-LPB ensemble simulations over South America and possible implications for climate projections. Clim Res. 2016;68:117-36.

54. Curry JA, Lynch AH. Comparing Arctic regional climate models. Eos. 2002;83:87.

55. Rinke A et al. Evaluation of an ensemble of Arctic regional climate models: spatiotemporal fields during the SHEBA year. Clim Dyn. 2006;27:433-49.

56. Reckerman $\mathrm{M}$ et al. BALTEX - an interdisciplinary research network for the Baltic Sea region. Environ Res Lett. 2011;6:045205.

57. Artale $\mathrm{V}$ et al. An atmosphere-ocean regional climate model for the Mediterranean area: assessment of a present climate simulation. Clim Dyn. 2010;35:721-40.

58. Sevault $\mathrm{F}$ et al. A fully coupled Mediterranean regional climate system model: design and evaluation of the ocean component for the 1980-2012 period. Tellus A. 2014;66:23967.

59. Turuncoglu UU, Giuliani G, Elguindi N, Giorgi F. Modeling the Caspian Sea and its catchment area using a coupled regional atmosphere-ocean model (RegCM-ROMS): model design and preliminary results. Geosci Model Dev. 2013;6:283-99.

60. Ratnam JV, Giorgi F, Kaginalkar A, Cozzini S. Simulation of the Indian monsoon by the RegCM3-ROMS regional coupled model. Clim Dyn. 2009;33:119-39.

61. Huth R. Statistical downscaling for central Europe. Evaluation of methods and potential predictors. Clim Res. 1999;13:91-103.

62. Salathe E. Comparison of various precipitation downscaling methods for the simulation of streamflow in a rainshadow river basin. Int J Climatol. 2003;23:887-901.

63. Goodess CM et al. An intercomparison of statistical downscaling methods for Europe and European regions: assessing their performance with respect to extreme weather events and the implications 
for climate change applications. In: CRU Research Reports. Norwich: Climatic Research Unit, University of East Anglia; 2010.

64. Burger G, Murdock TQ, Werner AT, Sobie SR, Cannon AJ. Downscaling extremes - an intercomparison of multiple statistical methods for present climate. J Clim. 2012;25:4366-88.

65. Benestad RE. A new global set of downscaled temperature scenarios. J Clim. 2011;24:2080-98.

66. Hayhoe K, VanDorn J, Croley II T, Schlegal N, Wuebbles D. Regional climate change projections for Chicago and the US Great Lakes. J Great Lakes Res. 2010;36:7-21.

67. Pryor SC, Barthelmie RJ. Hybrid downscaling of wind climates over the eastern USA. Environ Res Lett. 2014;9:AR 024013.

68. Maraun D. Bias correction, quantile mapping, and downscaling: revisiting the inflation issue. J Clim. 2013;26:2137-43.

69. Murphy JM. An evaluation of statistical and dynamical techniques for downscaling local climate. J Clim. 1999;12:2256-84.

70. Ayar PV et al. Intercomparison of statistical and dynamical downscaling models under the EURO- and MED-CORDEX initiative framework: present climate evaluations. Clim Dyn. 2016;46: 1301-29.

71. Huth R, Miksovsky J, Stepanek P, Belda M, Farda A, Chladova Z, Pisoft P. Comparative validation of statistical and dynamical downscaling models on a dense grid in central Europe: temperature. Theor Appl Climatol. 2015;120:533-53.

72. Schmidli J, Goodess CM, Frei C, Haylock MR, Hundecha Y, Ribalaygua J, Schmith T. Statistical and dynamical downscaling of precipitation: an evaluation and comparison of scenarios for the European Alps. J Geophys Res Atmos. 2007;112:D04105.

73. Fox-Rabinowitz M, Cote J, Dugs B, Deque M, McGregor J. Variable resolution general circulation models: stretched grid model intercomparison project (SGMIP). J Geophys Res - Atmos. 2006;111:D16104.

74. Sakaguchi K, Leung LR, Zhao C, Yang Q, Lu J, Hagos S, Rauscher SA, Dong L, Ringler TD, Lauritzen PH. Exploring a multiresolution approach using AMIP simulations. J Clim. 2015;28:5549-74.

75. Dee DP et al. The ERA-interim reanalysis: configuration and performance of the data assimilation system. Q J R Meteorol Soc. 2011;137:553-97.

76. Nikulin G et al. Precipitation climatology in an ensemble of CORDEX-Africa regional climate simulations. J Clim. 2012;25: 6057-78.

77. Jacob D et al. EURO-CORDEX: new high resolution climate change projections for European impact research. Reg Environ Chang. 2013;14:563-78.

78. Ruti $\mathrm{P}$ et al. MED-CORDEX initiative for Mediterranean climate studies. Bull Am Meteorol Soc. 2016. doi:10.1175/BAMS-D14.00176.1.

79. Koenigk T, Berg P, Döscher R. Arctic climate change in an ensemble of regional CORDEX simulations. Polar Res. 2015;34:AR 24603.
80. Ghimire S, Choudary A, Dimri AP. Assessment of the performance of CORDEX-South Asia experiments for monsoonal precipitation over the Himalayan region during present climate: part I. Clim Dyn. 2016. doi:10.1007/s00382-015-2747-2.

81. Park $\mathrm{C}$ et al. Evaluation of multiple regional climate models for summer climate extremes over East Asia. Clim Dyn. 2016;46: 2469-86.

82. Juneng L et al. Sensitivity of Southeast Asia rainfall simulations to cumulus and air-sea flux parameterizations in regCM4. Clim Res. 2016:69:59-77.

83. Sanchez E et al. Regional climate modeling in CLARIS-LPB: a concerted approach towards twenty first century projections of regional temperature and precipitation over South America. Clim Dyn. 2015;45:2193-212.

84. Cerezo-Mota R et al. CORDEX-NA: factors inducing dry/wet years on the North American monsoon region. Int J Climatol. 2016;36:824-36.

85. Diro GT et al. Tropical cyclones in regional climate change projections with RegCM4 for the CORDEX central America domain. Clim Chang. 2014;125:79-94.

86. Bucchignani E, Cataneo L, Panitz HJ, Mercogliano P. Sensitivity analysis with the regional climate model COSMO-CLM over the CORDEX-MENA domain. Meteorology and Atmopsheric Physics. 2016;128:73-95.

87. Ozturk T, Altinsoy H, Turkes M, Kurnaz LM. Simulation of temperature and precipitation climatology for the Central Asia CORDEX domain using RegCM4.0. Clim Res. 2012;52:63-76.

88. Gualdi $\mathrm{S}$ et al. The CIRCE simulations. Regional climate change projections with realistic representation of the Mediterranean Sea. Bull Am Meteorol Soc. 2013;94:65-81.

89. Nabat $\mathrm{P}$ et al. Direct and semi-direct aerosol radiative effect on the Mediterranean climate variability using a couple regional climate system model. Clim Dyn. 2015;44:1127-55.

90. Moss RH et al. The next generation of scenarios for climate change research and assessment. Nature. 2010;463:747-56.

91. Isotta FA et al. The climate of daily precipitation in the Alps: development and analysis of a high resolution gridded dataset from pan-Alpine rain-gauge data. Int J Climatol. 2014;34:1657-75.

92. Prein AF et al. Precipitation in the EURO-CORDEX 0.11 degrees and 0.44 degrees simulations: high resolution, high benefits? Clim Dyn. 2016;46:383-412.

93. Kendon EJ et al. Heavier summer downpours with climate change revealed by weather forecast resolution models. Nat Clim Chang. 2014;4:570-6.

94. Prein $\mathrm{AF}$ et al. A review on regional convection-permitting climate modeling: demonstrations, prospects and challenges. Rev Geophys. 2015;53:323-61.

95. Lafon T, Dadson S, Buys G, Prudhomme G. Bias correction of daily precipitation by a regional climate model: a comparison of methods. Int J Climatol. 2013;33:1367-81.

96. CORDEX News 2015. http://cordex.org/index.php?option=com content \&view $=$ article $\& i d=245$ : esd - workshop-jun $15-$ report\&catid $=117 \&$ Itemid $=735$ 